\title{
Comparing Unsteady Modeling Approaches of Surges Caused by Sudden Air Pocket Compression
}

\author{
Thomas M. Hatcher, ${ }^{1}$ Ahmad Malekpour, ${ }^{2}$ José G. Vasconcelos ${ }^{1}$ and Bryan W. Karney \\ ${ }^{1}$ Auburn University, Auburn, Alabama; ${ }^{2}$ University of Toronto, Toronto, Ontario.
}

Received 2014-06-06, accepted 2015-04-03, published 2015-04-17.

\begin{abstract}
The entrapment and compression of air in closed conduits is a relevant problem in pipeline systems that experience unsteady flow regimes. Severe surging resulting from large air compressibility leads to failures, structural damage and other operational issues. Various studies have been performed on the topic, most of which simplified the flow equations by adopting a lumped inertia approach to simulate the unsteady water flow, an implementation of the ideal gas law, momentum equation and air-water continuity equations. The modeling benefits of a discretized approach, such as the method of characteristics (MOC), to simulate the water phase have not been sufficiently investigated. To address this knowledge gap, this paper compares an MOC and a lumped inertia model in adverse pipe slope conditions involving sudden air pocket compression caused by the closure (partial or total) of a downstream knife gate valve. In laboratory experiments air pressures and flow rates were measured during various sudden air compression events, serving to assess the accuracy of each modeling approach. Results of the comparison indicate that the two hydraulic models have comparable accuracy for partial valve closure cases. For total valve closures, the models are comparable for smaller surge events but significantly diverge when maximum $H / D$ values exceed 60 to 80 . This feature did not depend on the pipe $L / D$ ratio for the proposed experiments, but the magnitude of error was affected by this ratio, specifically when the air pocket volume was less than unity. Additional experiments are needed to better assess the effects of the pipeline $L / D$ ratio and other geometrical parameters, such as slope, on peak surge predictions.

Keywords: unsteady flows, surges, air pockets, numerical models.
\end{abstract}

\section{Introduction}

The issue of air pocket entrapment in closed conduits is relevant both in the context of water and sewerage transmission mains and in the problem of rapid filling of stormwater sewers and tunnels. Pozos et al. (2010) pointed that mechanisms for air pocket entrapment in transmission mains include residual air from priming operations, entrainment of air at high points of pipelines, and at intake vortices, among others. In the context of stormwater systems, Vasconcelos and Wright (2006) presented a list of various mechanisms, which included shear-force instabilities, open channel bore reflections from system boundaries, and interface breakdowns of pipe-filling bores, among others. Particularly in the case of stormwater systems, it is impossible to predict the exact location and size of entrapped air pockets due to the unpredictable nature of inflows that enter the system through inflow structures and dropshafts. Thus, it becomes important to assess the potential for surges in such systems, and design systems accordingly.
Martin (1976) presented the pioneer work on air-water interactions during compression-expansion cycles. The low frequency pressure oscillations had magnitudes far exceeding the pressures that drove the flow into the air pockets. This feature is caused by the high compressibility of air, which varies with the air pocket volume and inertia of the water column compressing the air. Other studies expanded on this work. Zhou et al. (2002) presented experimental results of various air compressions with ventilation orifices of varying sizes. Lee (2005) included a more accurate thermodynamics description for the air phase during the compression and expansion cycles. De Martino et al. (2008) performed further experiments varying pocket lengths and driving pressure heads. Vasconcelos and Leite (2012) presented results from experiments in which air pockets were entrapped by sudden maneuver of a valve blocking a free discharge at the end of a pipe, aiming to produce a condition that is more representative of air entrapment in stormwater systems.

Some of these investigations also involved the numerical modeling of flows. These models were often of a lumped inertia type, with the air phase represented by an implementation of the

Hatcher, T.M., A. Malekpour, J. Vasconcelos and B. Karney. 2015. "Comparing Unsteady Modeling Approaches of Surges Caused by Sudden Air Pocket Compression." Journal of Water Management Modeling C392. doi: 10.14796/JWMM.C392.

(c) CHI $2015 \quad$ www.chijournal.org ISSN: 2292-6062 
continuity equation and the ideal gas law. While lumped inertia models are appropriate to describe the conditions reported in experiments, discretized solutions have also been used. Such models have the advantage of accounting for pipe elasticity and fluid compressibility. Reddy et al. (2010) and Zhou et al. (2011) are examples of studies that implemented the simulation of rapid flows with air compression using the method of characteristics (MOC). Vasconcelos and Wright (2009) applied the TPA model to simulate the rapid filling considering air pocket compression. Malekpour and Karney (2014) also indicated that after a certain pipeline lengths surge predictions from lumped inertia models are largely overestimated.

Most these studies have not focused on a direct systematic comparison between lumped inertia and distributed modeling approaches. Moreover, in most cases a comparison between numerical models and experiments did not involve different pipe diameters to assess any potential scale effects of the findings. Such important questions need to be addressed to advance our modeling efforts in simulating extreme flow conditions caused by air-water interactions in closed conduit flows. Accurate peak pressure predictions can be essential in reducing cost and meeting safety requirements, and the appropriate numerical model for such applications is not always clear.

\section{Objectives}

This work aims to assess and compare the accuracy of MOC and lumped inertia models in simulating surging caused by air pocket compression. Systematic experiments involving surges caused by air compression were performed following the work of Vasconcelos and Leite (2012). A lumped inertia (RC) and discretized numerical model (MOC) were formulated and the model predictions were compared to the experimental measurements in a $53 \mathrm{~mm}$ diameter $(D=53 \mathrm{~mm}$ ) pipe. Model predictions were also compared to the experimental results from Vasconcelos and Leite (2012) obtained with a larger $D=102 \mathrm{~mm}$ pipe. The conditions tested involved varying air pocket volumes, initial flow rates and degrees of valve closures. The comparison between results included pressure and flow variation following valve maneuvers.

\section{Methodology}

\subsection{Experiments}

To study a large range of flow parameters for surges caused by sudden air pocket compression, an experimental apparatus was constructed in the Hydraulic Research Laboratory at Auburn University. This apparatus was constructed with two different pipes ( $D=53 \mathrm{~mm}$ and $D=102 \mathrm{~mm}$ ) and consisted of a variable speed pump recirculation system. There was a $0.66 \mathrm{~m}^{3}$ upstream reservoir (plan area $=0.50 \mathrm{~m}^{2}$ ) connected to a $10.7 \mathrm{~m}(\mathrm{D}=53 \mathrm{~mm})$ or $12.0 \mathrm{~m}(\mathrm{D}=102 \mathrm{~mm})$ clear PVC pipeline.

The $\mathrm{D}=52 \mathrm{~mm}$ pipeline experiments were recently conducted to facilitate this work while the $D=102 \mathrm{~mm}$ experiments were performed by Vasconcelos and Leite (2012). They did not present pressure or velocity hydrographs for adverse slopes, which is a major focus of this work. For both diameter experiments, there was a small pipe segment ( $1 \mathrm{~m}$ long) connecting the upstream reservoir to the main pipeline. The diameter of this small pipe was $53 \mathrm{~mm}$ and $102 \mathrm{~mm}$ for $\mathrm{D}=102 \mathrm{~mm}$ and $\mathrm{D}=53 \mathrm{~mm}$ experiments, respectively. This expansion or contraction in pipe cross-sections had little impact on surge results.

The main pipeline was setup with a constant adverse slope of $2.0 \%$ as displayed in Figure 1. The slope was established with the aid of a laser level; the estimated accuracy was $\pm 0.1 \%$. At the downstream end of the apparatus, a knife gate valve was added with the same diameter as the pipeline, and free discharge conditions existed prior to valve maneuvering. The pipeline was anchored both laterally and longitudinally.

Sufficiently far upstream from each air pocket, a Nortek Vectrino MicroADV was installed to determine flow velocities with a sampling frequency of $30 \mathrm{~Hz}$. The ADV was placed $3.0 \mathrm{~m}$ from the upstream end of the pipeline for $D=102 \mathrm{~mm}$ experiments as described in Vasconcelos and Leite (2012). In experiments with the $D=53 \mathrm{~mm}$ pipeline, this MicroADV probe was installed in the short $(D=102 \mathrm{~mm}$ ) pipe segment $1.0 \mathrm{~m}$ from the upstream reservoir. The continuity equation was used to relate velocities measured in the $102 \mathrm{~mm}$ diameter segment to the velocities in the $53 \mathrm{~mm}$ pipeline. These velocity results were validated with reservoir emptying experiments in which the reservoir water level was measured over time to confirm the validity of this approach.

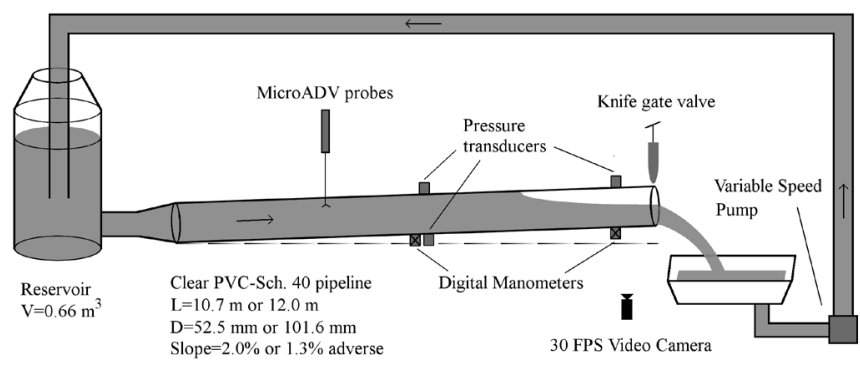

Figure 1 Schematic diagram of entrapped air pocket experiments used in this work.

For all experiments, three pressure transducers (MEGGIT-ENDEVCO 8510-50C, maximum pressure $345 \mathrm{kPa}, 0.4 \%$ accuracy) were used sampling at a frequency of $100 \mathrm{~Hz}$. A transducer pair was installed at the pipe crown and invert near the middle of the pipe, and the third at the pipe crown $\sim 30 \mathrm{~cm}$ from the downstream end of the pipe, which was used to measure the pressure of the air pocket that formed upon valve closure. In this work the focus was on air pressure measurements (the downstream pressure transducer), but the other transducer results were used for experimental validation.

Before the valve was closed, steady flow was established in the pipeline, so pressurized flow existed in the upstream end of the pipe and free surface flow at the downstream end. Flow was recirculated through a downstream reservoir that was connected 
to a variable speed pump. Water was constantly flowing into the upstream reservoir from this pump. The experimental procedure essentially follows Vasconcelos and Leite (2012) experiments:

1. Adjust the variable speed pump and flow control valves to achieve the desired relative flow rate $\left(Q^{*}\right)$ and allow the flow to reach steady state; the flow was assumed steady when the water level in the reservoir and the air pocket length were constant;

2. Measure the arc length of the downstream air pocket at several stations: there was an average of fourteen measurements which varied in length based on the pocket length (Lair);

3. Record the pressure head with three manometers setup along the apparatus: located at the reservoir, pipeline midpoint, and the downstream end. This data was used to extrapolate results from the pressure transducers;

4. Start recording with a 30 FPS digital camcorder (1920 x 1080 resolution) that was set up at the downstream end of the pipe. The camera was used to observe flow features for the air pocket and to determine the valve closure time;

5. Activate data logging for MicroADV and pressure transducers;

6. Setup additional lighting for the video camera and close the knife gate valve as quickly as possible (partially or completely) without causing noticeable pipe oscillations;

7. For partial closures, completely shut the valve and allow the water level to rise $\sim 20 \%$ to $40 \%$ in the upstream reservoir, which increased the accuracy of pressure calibrations;

8. Shutoff the pump, record the updated manometer readings and stop data recording for the MicroADV and pressure transducers.

Each experimental condition was repeated at least two times until consistency in results was obtained. The range of experimental values is presented in Table 1; parameters were normalized in terms of the pipeline diameter $D$ and flow rate $Q$ :

Table 1 Range of experimental conditions tested in numerical modeling studies.

\begin{tabular}{ll}
\hline \multicolumn{1}{c}{ Parameter } & \multicolumn{1}{c}{ Range tested } \\
\hline Flow rate: $Q^{*}=Q / \sqrt{g D^{5}}$ & At least four values for each type of closure \\
& $Q^{*}=0.08-0.50$ \\
Air pocket volume: $V a^{*}=V I D^{3}$ & At least four values for each type of closure \\
& $V a^{*}=0.32-6.59$ \\
Pipeline slope & $S O=1.3 \%$ adverse for $D=101.6 \mathrm{~mm}$ \\
& $S O=2.0 \%$ adverse for $D=52.5 \mathrm{~mm}$ \\
\hline
\end{tabular}

\subsection{Rigid Column (RC) Model}

The lumped inertia, rigid column (RC) model formulation follows the work of Vasconcelos and Leite (2012) focusing on adverse slope conditions. Thus, the primary addition for the lumped inertia model in this work is the weight component, which is incor- porated in the momentum expression. The model formulation is 1D in which the focus is on pressure and discharge variations over time. Moreover, the RC model can predict peak pressures relatively accurately despite the highly complex nature of air-water flow interactions following the sudden entrapment of air (Vasconcelos and Leite 2012).

The convenience of the proposed method is based on the simplicity in applying these air-water interactions in tunnel flow models without adding excessive computational effort. The two phase lumped inertia model (Wylie and Streeter 1993) uses ideas from Li and McCorquodale (1999) and Zhou et al. (2002). It consists of three primary ordinary differential equations (ODEs): a momentum equation for the water phase, a continuity equation for the air phase, and the ideal gas law.

$$
\begin{aligned}
& \frac{d Q}{d T}=\frac{g A}{L}\left[H_{r e s}-\left(H_{a i r}-H_{a t m}\right)+L S_{0}-\left(\left(f \frac{L}{D}+K_{\text {loss }}\right) \frac{Q|Q|}{2 g A^{2}}\right)\right] \\
& \frac{d V_{a}}{d T}=-Q+C_{d} A_{\text {orif }} \sqrt{2 g\left(H_{\text {air }}-H_{\text {atm }}\right)} \\
& \frac{d H_{\text {air }}}{d T}=k \frac{H_{\text {air }}}{V_{a}} \frac{d V_{a}}{d T}
\end{aligned}
$$

where:

$$
\begin{aligned}
T & =\text { time, } \\
g & =\text { acceleration due to gravity, } \\
A & =\text { cross-sectional area of pipe, } \\
L & =\text { length of rigid column, } \\
H_{\text {res }} & =\text { pressure head at the pipeline upstream end } \\
H_{\text {air }} & =\text { air phase pressure head, } \\
H_{\text {atm }} & =\text { atmospheric pressure head, } \\
f & =\text { Darcy-Weisbach friction factor, } \\
D & =\text { pipe diameter, } \\
\mathrm{K}_{\text {loss }} & =\text { summation of local losses, } \\
V_{a} & =\text { air pocket volume } \\
C_{d} & =\text { discharge coefficient, } \\
A_{\text {orif }} & =\text { discharge area at knife gate valve, and } \\
k & =\text { polytropic coefficient. }
\end{aligned}
$$

The momentum equation expresses water flow rate $(Q)$ upstream from the air pocket region. Rather than disregard the water mass initially in free-surface flow conditions under the downstream air cavity, the $\mathrm{RC}$ model accounts for the overall water mass through the expression $L=\left(L_{\text {pipe }} A-V_{a}\right) / A$, with $L_{\text {pipe }}$ as the length of the pipeline. Another important assumption of the proposed model is that an orifice-like equation that depends on the air pressure upstream of the gate can describe the outflow rate (Equation 2).

The set of ODEs was solved with a fourth order RungeKutta method as presented in Press et al. (1989). For the air compression, $k$ was assumed to be equal to 1.2 as in Vasconcelos and Leite (2012). The volume of air was calculated within the RungeKutta method by rearranging the terms in Equation 3: 


$$
d V_{a}=\frac{1}{k} \frac{V_{a}}{H_{\text {air }}} d H_{\text {air }}
$$

It is acknowledged that there are limitations in assuming a constant friction factor in this model. A number of relevant factors may play a role in defining the actual energy damping of these flows including frequency dependent friction (Wylie and Streeter 1993), viscoelastic effects in plastic pipes (Soares et al. 2008) and thermofluid dynamics effects (Lee 2005). While numerical modeling results indicate that observed energy damping is stronger than what is obtained using a constant friction factor, this aspect requires investigations that are outside the scope of this work.

Unsteady water levels are accounted for in the upstream reservoir, which receives a constant flow of water. For the initial conditions in this model, $Q$ and $H_{\text {res }}$ were obtained from experimental measurements, and $H_{\text {air }}$ was assumed equal to $H_{\text {atm }}$. As previously mentioned, $V_{a}$ was calculated by numerical integration of the dry perimeter of the air pocket at the pipe crown at regular intervals.

The RC modeling approach was selected because it succeeded in providing estimates for maximum and minimum pressures involving air compression in previous studies. This success is probably explained by the dominant effect of the air pocket in determining unsteady flow pressures when compared to other problem features such as pipe wall elasticity and the water compressibility. This enables the application of the lumped inertia approach with good accuracy.

The discharge coefficient was calibrated from experiments after valve closure once steady state flow conditions had developed ( $5 \mathrm{~s}$ to $10 \mathrm{~s}$ ). For $\mathrm{D}=102 \mathrm{~mm}$ pipes, there was little variation in discharge coefficient calculations between experiments, and $C d=0.47$ for $81 \%$ valve closure and $C d=0.33$ for $89 \%$ valve closure. However, applying a constant $C d$ for $D=53 \mathrm{~mm}$ experiments resulted in inaccurate pressure predictions. The maximum pressures measured in experiments were underestimated by $\sim 50 \%$.

Numerical calibration of $C d$ for maximum pressures resulted in values of approximately 0.40 that were independent of the degree of valve closure for the partial closures tested in this work. $C d=0.40$ is significantly smaller than values calibrated from experiments, which averaged 0.63 with little variation. As a result, implementing $C d=0.40$ in the numerical models caused overestimation of steady state flow rates. To overcome this error, an ad hoc solution was utilized in this work for $D=53 \mathrm{~mm}$ experiments in which a linear expression was developed for $C d$. This expression is a function of the numerical calibration and experimental calibration of $C d$ as follows:

$$
C d=0.4-\frac{\left(0.4-C d_{f}\right)\left(R e_{\max }-R e\right)}{R e_{\max }-R e_{f}}
$$

where:

$$
C d=\text { discharge coefficient calculated each time step, }
$$

$$
\begin{aligned}
& C d_{f}=\text { final discharge coefficient calibrated from } \\
& \text { experiments after valve closure once steady } \\
& \text { state conditions developed, } \\
& R e=\text { Reynolds number calculated each time step, } \\
& R e_{f}=\text { final Reynolds number calibrated from } \\
& \text { experiments after valve maneuver and after } \\
& \text { steady state conditions developed, and } \\
& R e_{\text {max }}=\text { Reynolds number associated with the maximum } \\
& \text { velocity, occurring before valve closure. }
\end{aligned}
$$

Setting the maximum $C d=0.40$ worked well for $D=53 \mathrm{~mm}$ pipes with $81 \%$ and $89 \%$ closures but would have to be recalibrated for different systems. A possible explanation in discharge coefficient discrepancies between $D=53 \mathrm{~mm}$ and $D=102 \mathrm{~mm}$ experiments is the geometric differences between the valves. Moreover, the length between the bottom of the valve obstruction and the bottom of the pipe is different for a specified degree of closure in $D=53 \mathrm{~mm}$ and $\mathrm{D}=102 \mathrm{~mm}$ experiments, thus affecting the energy losses at the valve. In addition, $C d$ expressions were derived from the steady state Bernoulli equation, so the most appropriate $C d$ implementation for transient events is not fully understood.

\subsection{Method of Characteristics (MOC) Model}

One-dimensional transient flow in closed conduits is governed by two partial differential equations referred to as the momentum and continuity equations (Wylie and Streeter 1993; Chaudhry 1987), respectively:

$$
\begin{aligned}
& \frac{\partial V}{\partial t}+g \frac{\partial H}{\partial x}+\frac{f}{2 D}|V| V=0 \\
& \frac{\partial H}{\partial x}+\frac{a^{2}}{g} \frac{\partial V}{\partial x}=0
\end{aligned}
$$

where:

$$
\begin{aligned}
V & =\text { velocity } \\
H & =\text { piezometric head, } \\
a= & \text { elastic wave velocity, and } \\
x, t= & \text { the independent variables of distance and time, } \\
& \text { respectively. }
\end{aligned}
$$

Although analytical solutions can be provided in trivial cases, numerical methods are inevitable in complicated systems with complex boundary conditions. Among a variety of available numerical methods, the method of characteristics is one of the most powerful tools that have been widely used for solving water hammer equations.

This method transforms the governing equations into four ordinary differential equations that can be integrated through the paths called the characteristic curves to find the dependent variables at specific distances and times. The characteristic equations for the water hammer equations are as follows (Wylie and Streeter 1993).

$$
C^{+}: \frac{g}{a} \frac{d H}{d t}+\frac{d V}{d T}+\frac{f V|V|}{2 D}=0
$$




$$
\begin{aligned}
& \frac{d x}{d t}=+a \\
& C^{-}:-\frac{g}{a} \frac{d H}{d t}+\frac{d V}{d T}+\frac{f V|V|}{2 D}=0 \\
& \frac{d x}{d t}=-a
\end{aligned}
$$

These equations show that along the positive characteristic $\left(C^{+}\right)$and negative characteristic $\left(C^{-}\right)$lines, Equations 8 and 10, named compatibility equations, respectively govern the flow. At the intersection point where the two characteristic lines meet each other, the independent variables $(V$ and $H$ ) can be calculated by simultaneous solution of the equations.

The finite difference solution of the compatibility equations can be made based on a finite difference computational mesh depicted in Figure 2.

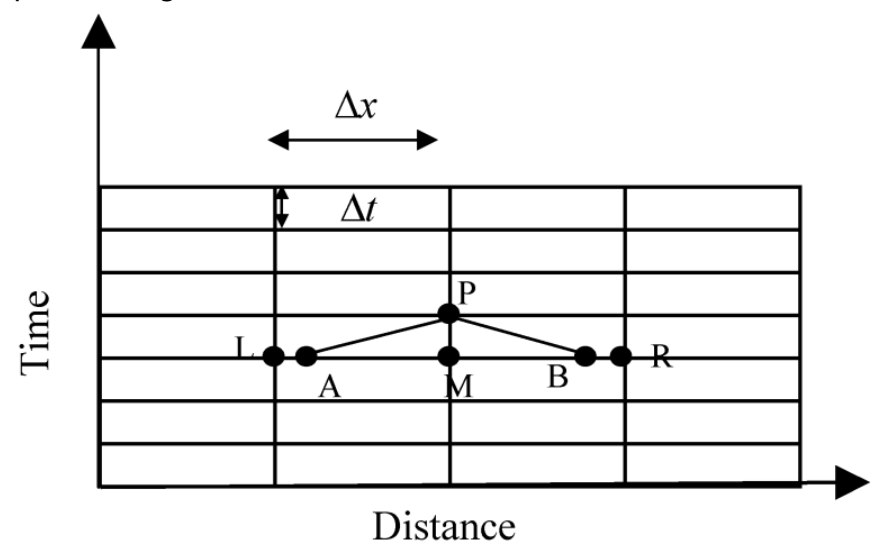

Figure 2 Characteristics lines in a typical finite difference computational mesh.

This figure shows that the unknown variables at the current time for a given grid point $(P)$ can be calculated using the information of known points $A$ and $B$. For the sake of generality, it is assumed that the characteristic lines do not meet the time line exactly at grid points where the information is stored. The discrete form of the compatibility equations can then be written as follows (Wylie and Streeter 1993).

$$
\begin{aligned}
& C^{+}: H_{P}=C_{1}-C_{2} Q_{P} \\
& C^{-}: H_{P}=C_{3}+C_{4} Q_{P}
\end{aligned}
$$

where:

$$
\begin{aligned}
& C_{1}=H_{A}+\frac{a}{g A} Q_{A} ; \text { and } C_{2}=\frac{a}{g A}+\frac{f \Delta x}{2 g D A^{2}}\left|Q_{A}\right| \\
& C_{3}=H_{B}-\frac{a}{g A} Q_{B} ; \text { and } C_{4}=\frac{a}{g A}+\frac{f \Delta x}{2 g D A^{2}}\left|Q_{B}\right|
\end{aligned}
$$

$Q_{A^{\prime}} Q_{B^{\prime}} H_{A}$ and $H_{B}$ are not stored and should be linearly interpolated from the grid point's information by using the following equations:

$$
Q_{A}=Q_{M}+\frac{a \Delta t}{\Delta x}\left(Q_{L}-Q_{M}\right) ; \text { and } H_{A}=H_{M}+\frac{a \Delta t}{\Delta x}\left(H_{L}-H_{M}\right)
$$

$$
Q_{B}=Q_{M}+\frac{a \Delta t}{\Delta x}\left(Q_{R}-Q_{M}\right) ; \text { and } H_{B}=H_{M}+\frac{a \Delta t}{\Delta x}\left(H_{R}-H_{M}\right)(17)
$$

where:

$\Delta x, \Delta t=$ space and time increments, respectively.

Looking at Equations 12 and 13, both equations are linear and are solved simultaneously to determine $H$ and $Q$ at point $P$.

The time step is calculated through using the well-known Courant-Friedrich-Lewy (CFL) stability criterion (Chaudhry 1987). For a system with a single pipe, the time step can be easily calculated by $\Delta t=\Delta x / a$ where the Courant number is set to one. In complex pipe systems having multiple pipes, each pipe may require its own time steps as indicated by the relation $\Delta t=L /(a N)$, where $L$ is the length of the pipe line, $a$ is the wave velocity, $N$ is an integer showing the number of division for the pipe, and $\Delta x$ represents the reach length.

All unknowns in the computational domains except those at boundary nodes can be explicitly determined by using the aforementioned calculation procedure. In order to calculate the unknowns at boundary nodes, more information is required. In this work a constant water level reservoir is considered for the upstream boundary condition, as shown in Figure 3.

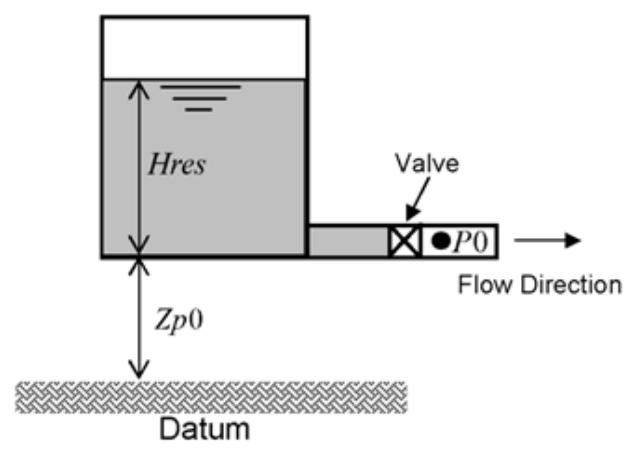

Figure 3 Schematic diagram of the upstream boundary.

In order to calculate unknowns at the upstream boundary point $(P 0)$, the following equations are considered: the negative characteristics equation and energy equation, respectively.

$$
\begin{aligned}
& C_{3} C^{-}:\left(H_{P 0}\right)_{t+\Delta t}=C_{3}+C_{4}\left(Q_{P 0}\right)_{t+\Delta t} \\
& H_{r}+Z_{P 0}=\left(H_{P 0}\right)_{t+\Delta t}+\left(1+K_{V}\right) \frac{\left(Q_{P 0}\right)_{t+\Delta t}{ }^{2}}{2 g A^{2}}
\end{aligned}
$$

where:

$$
\begin{aligned}
Q_{P 0^{\prime}} H_{P O}= & \text { instantaneous flow discharge and piezometric } \\
& \text { head in the upstream boundary respectively, } \\
H_{r}= & \text { reservoir depth, and } \\
K_{V}= & \text { valve head loss coefficient. }
\end{aligned}
$$

By simultaneous solution of the above equations, $\left(Q_{p o}\right)_{t+\Delta t}$ is calculated as follows:

$$
\left(Q_{P 0}\right)_{t+\Delta t}=0.5\left(-C C_{1}+\sqrt{C C_{1}^{2}-4 C C_{2}}\right)
$$


In the case of reverse flow, the sign of the second term on the right hand side of Equation 20 becomes negative and the following equation is used:

$$
\left(Q_{P 0}\right)_{t+\Delta t}=0.5\left(C C_{1}-\sqrt{C C_{1}^{2}+4 C C_{2}}\right)
$$

where:

$$
\begin{aligned}
& C_{3}=\left(H_{1}\right)_{t}-\frac{a}{g}\left(Q_{1}\right)_{t} ; \text { and } C_{4}=\frac{a}{g A}+\frac{f \Delta x}{2 g D A^{2}}\left|\left(Q_{1}\right)_{t}\right| \\
& C C_{1}=\frac{2 g A^{2} C_{4}}{1+K_{V}} ; \text { and } C C_{2}=\frac{2 g A^{2}\left(C_{3}-H_{r e s}-Z_{P 0}\right)}{1+K_{V}}
\end{aligned}
$$

To calculate the unknowns at the downstream boundary node consider Figure 4.

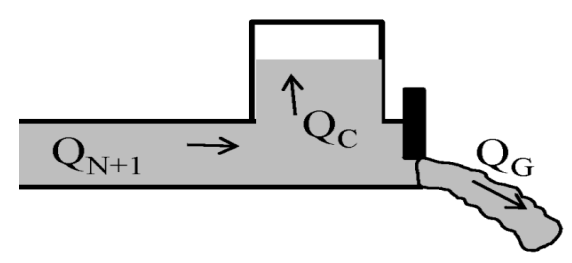

Figure 4 Schematic diagram of the downstream boundary.

The following expressions that include the continuity equation, positive characteristic equation, energy equation and the equation of state for air (Equation 3) are computed at the downstream BC:

$$
\begin{aligned}
& \left(Q_{N+1}\right)_{t+\Delta t}=Q_{C}+Q_{G} \\
& C^{+}:\left(H_{N+1}\right)_{t+\Delta t}=\left(H_{\text {air }}\right)_{t+\Delta t}-H_{a t m}+Z_{N+1}=C_{1}-C_{2}\left(Q_{N+1}\right)_{t+\Delta t} \\
& Q_{G}=C_{d} A_{\text {orif }} \sqrt{2 g\left(\left(H_{\text {air }}\right)_{t+\Delta t}-H_{\text {atm }}\right)} \\
& Q_{C}=\frac{d V_{a}}{d t}
\end{aligned}
$$

with

$$
C_{1}=\left(H_{N}\right)_{t}+\frac{a}{g A}\left(Q_{N}\right)_{t} ; \text { and } C_{2}=\frac{a}{g A}+\frac{f \Delta x}{2 g D A^{2}}\left|Q_{N}\right|
$$

where:

$$
\begin{aligned}
H_{\text {air }}= & \text { air pressure head, } \\
V_{a}= & \text { air volume, } \\
Q_{C}= & \text { air flow discharge, } \\
Q_{G}= & \text { liquid flow discharge, and } \\
Q_{N^{\prime}} Q_{N+1}= & \text { liquid flow discharge at computational nodes } N \\
& \text { and } N+1, \text { respectively. }
\end{aligned}
$$

This set of equations are simultaneously solved to obtain $H_{\text {air }}, V_{a^{\prime}} H_{N+1}, Q_{N+1}, Q_{C}$ and $Q_{G}$ at the current time step.

\section{Results and Discussion}

The numerical models were tested with partial valve closures ( $81 \%$ and $89 \%$ ) and total valve closures. Model performance was assessed based on peak pressure comparisons (maximum and minimum) for different experimental conditions. For partial valve closures, steady state results after valve closure were also analysed. Pressure and discharge hydrographs are provided for each condition in which the time parameter was normalized by $V_{a}^{1 / 3} / \sqrt{ }(g D)$. The experiments presented for each hydrograph were selected based on the following criteria: obtain a wide range of flow rates in which similar $V_{a}^{*}$ values were used for different diameter experiments.

Vasconcelos and Leite (2012) performed 1.3\% and 2.7\% adverse slope experiments, and their $1.3 \%$ slopes are presented in this work. A few numerical model tests were performed with $2.7 \%$ slopes to confirm that the results were not significantly dependent on slope since the air pocket volume changes accordingly. $2.0 \%$ adverse slopes were used with $\mathrm{D}=53 \mathrm{~mm}$ pipe experiments.

\subsection{Total Valve Closures}

Pressure hydrographs are displayed in Figure 5 for total valve closures in $D=53 \mathrm{~mm}$ and $D=102 \mathrm{~mm}$ pipe experiments. The air pocket volume and time parameters are $V_{a}^{*}=V_{a} / D^{3}$ and $T^{*}=T / V_{a}^{1 / 3} / \sqrt{ }(g D)$. For larger air pocket volumes, both numerical models predicted peak pressures relatively well compared to experiments. The maximum pressures were overpredicted by $20 \%$ to $25 \%$ for the $V_{a}^{*}=2.63$ and $V_{a}^{*}=3.70$ experiments presented in Figure 5. This accuracy generally decreased for smaller air pocket volumes. For the pressure decay, the $\mathrm{MOC}$ model was more accurate in comparison with the RC model, but both models performed poorly compared to experiments. Vasconcelos and Leite (2012) presented similar errors.
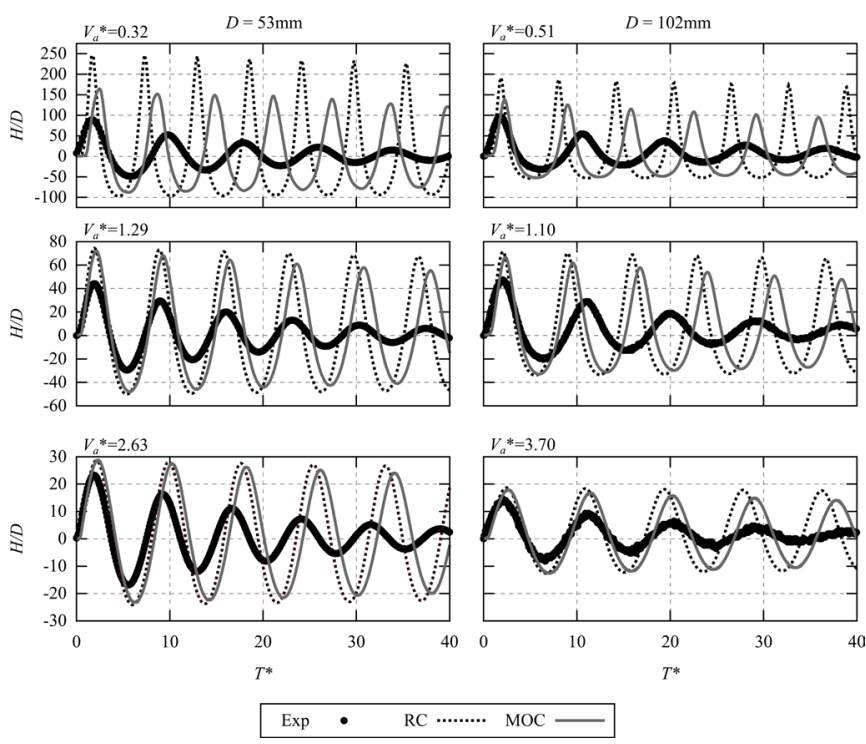

Figure 5 Pressure hydrographs for total valve closures. 
The pressure frequency predictions were also more accurate for experiments with larger air pocket volumes. For the smallest air pocket sizes tested $\left(V_{a}^{*}=0.3\right.$ to 0.5$)$, numerical modeling results diverged with the $\mathrm{MOC}$ model predicting pressure frequencies more accurately than the RC model. Some factors can help explain the discrepancy between numerical model results. One is the elasticity of PVC pipes, which can be captured by the MOC model but not by the RC model. This feature could also help to explain why the surge peaks with MOC are consistently attenuated compared to RC surge peaks. In addition, anchoring may not be perfect, especially for larger pressure linked to smaller air pocket volumes. A final point is the larger degree of uncertainty in measuring small air pocket volumes, which could also help explain why the measured pressure peaks are not so close to the model predictions in small $V_{a}^{*}$ conditions.

The total valve closure discharge hydrographs are presented in Figure 6 for both numerical models and experiments. The initial flow rate in experiments was used as an input parameter in the numerical models. For the minimum and maximum flow rates, the MOC model clearly outperforms the RC model for $V_{a}^{*}<1$ experiments but both numerical models performed well for larger air pocket sizes. The rigid column model significantly overpredicts peak flow rates for the smallest air pocket experiment. However, these errors were not as noticeable as in the maximum pressure comparison. Neither numerical model resolved the decay of inertial frequencies accurately as expected. Like the pressure oscillations, frequencies in discharge were simulated accurately only for experiments with larger air pocket volumes.
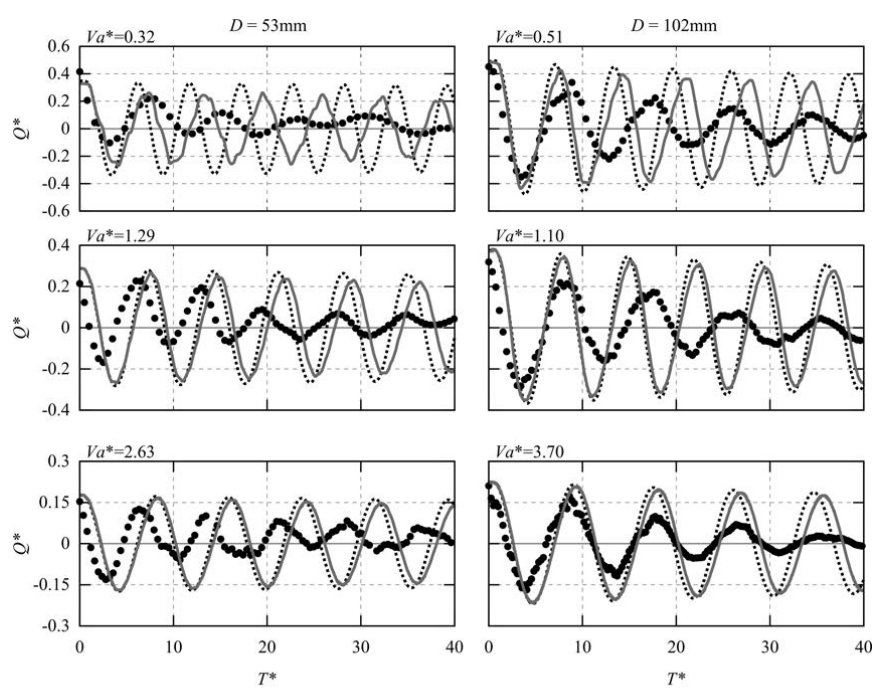

Exp $\quad$ RC $\cdots \cdots \cdots$

Figure 6 Flow rate hydrographs for total valve closures.

The peak pressures measured in experiments and predicted with numerical models are presented in Figure 7 for total valve closures. Both numerical models perform well when peak $H / D$ values are $<20$ and in which errors are $20 \%$ or less. For larger pressure ratios, the numerical model predictions tend to diverge from experiments. When maximum $H / D$ ratios are $\sim 40$, the $R C$ and $\mathrm{MOC}$ model predictions start to diverge from one another in which the RC model overpredicts maximum pressures more than the MOC model. For the largest pressure ratios tested $(H / D>80)$, the RC model significantly overpredicts measured and MOC predicted maximum pressures. Thus, the $\mathrm{RC}$ model is not recommended for $H / D>60$ to 80 scenarios.

Minimum pressure predictions were essentially the same for both numerical models. Similar to maximum pressures, both models performed well when $|H / D|<20$. For $H / D<-30$ scenarios, numerical model predictions diverge significantly from experiments for negative pressures (see Figure 7). Unlike maximum pressures, both numerical model predictions were similar for minimum pressures. This feature may be caused by smaller $|H / D|$ ratios measured in experiments for minimum pressures compared to maximum pressures. A small divergence is seen in Figure 7 between numerical model predictions when minimum pressure ratios $|H / D|>30$ as observed for maximum pressures with the RC model performing worse. Thus, it is possible that the same trend seen for maximum pressures exists for negative pressure predictions. A larger range of experimental conditions is needed with values of $H / D<-40$ to better understand negative pressure predictions.

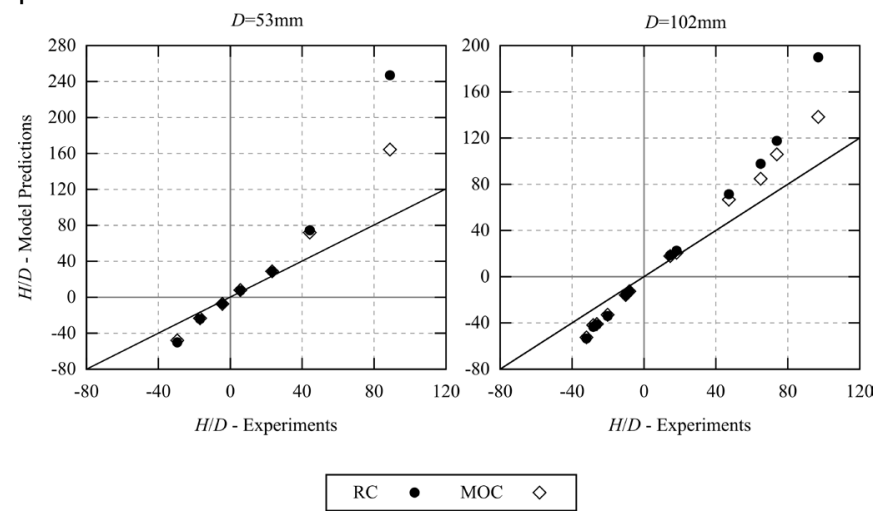

Figure 7 Peak pressure predictions compared to experiments for total valve closures.

The percent error between peak pressure predictions and experiments is fairly consistent for the pipe diameters as presented in Figure 7. When the maximum $H / D<50$, both numerical models overpredict peak pressures in experiments by approximately $40 \%$ for both diameters tested. However, when $H / D>80$, the RC model overpredicts experimental results by $180 \%$ and $100 \%$ for $D=53 \mathrm{~mm}$ and $\mathrm{D}=102 \mathrm{~mm}$ experiments, respectively. The results for the MOC model are $80 \%$ and $40 \%$, respectively. These increased errors for experiments with large surge pressures may also be caused by the difficulty in measuring the smaller air pockets, particularly for $D=53 \mathrm{~mm}$ experiments. Moreover, the pipe slopes were not the same for the different diameter experiments and thus the normalized air pocket volume $\left(V_{a}^{*}\right)$ differs. For example, $V_{a}^{*}$ was approximately 0.3 for $D=53 \mathrm{~mm}$ experiments and 0.5 for $D=102 \mathrm{~mm}$ experiments when $H / D \approx 90$. 


\subsection{Partial Valve Closures}

MOC and RC models were also tested with sudden $81 \%$ and $89 \%$ valve closures, and results were expected to improve in comparison with total closures. The $81 \%$ valve closures in Figure 8 are indeed more accurate than total closures, particularly for peak pressure predictions and pressure dissipation. For the partial valve closures, pressure damping is mostly caused by the energy release from the downstream side of the pipe, which is well accounted for in both models. However, in the case of full valve closure, friction is much more important and neither model simulates it well.

Both numerical models predicted similar results in most test cases. For a few experiments (e.g. $\mathrm{D}=102 \mathrm{~mm}, V_{a}^{*}=3.70$ ), the MOC model predicted the transition to steady state conditions post valve closure more quickly and at larger pressures than the RC model, due to small differences in energy loss calculations. This discrepancy was generally minor, and both models predicted steady state pressures accurately in most cases. The discrepancy between the numerical models at long times $\left(T^{*}<20\right.$ to 40$)$ was generally small; however, there were a few test cases where errors were larger than expected. The source of these errors is unclear, but the differences are suspected to be caused by differences in energy loss calculations performed at the downstream boundary upon reaching steady state conditions.

Steady state pressures were generally overpredicted by $30 \%$ or less except for the $\mathrm{D}=102 \mathrm{~mm}, V_{a}^{*}=3.70$ experiment shown in Figure 8, which represents the worst case. These results suggest that the numerical models perform better for the smaller diameter experiments, albeit the differences were minor.
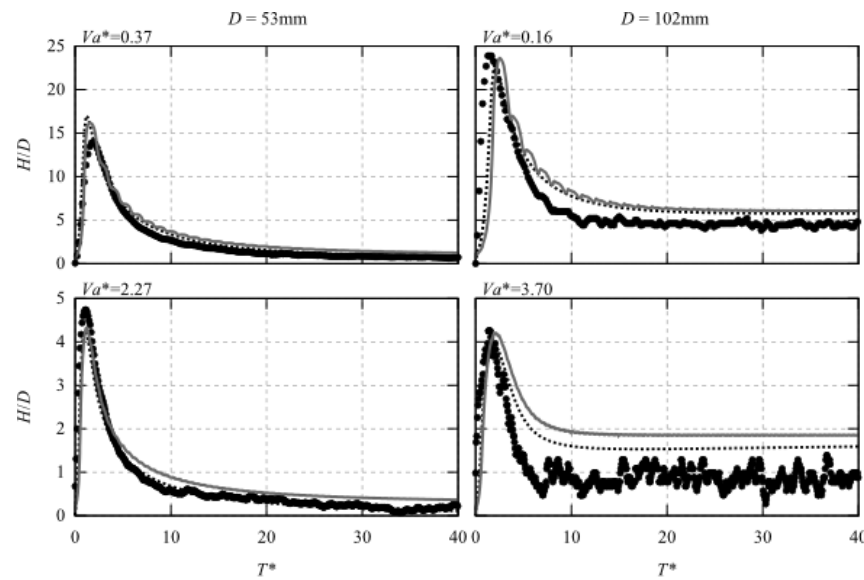

Exp - $\mathrm{RC} \cdots \cdots . . . . . . \mathrm{MOC}-$

Figure 8 Pressure hydrographs for $81 \%$ valve closures.

The discharge hydrographs for $81 \%$ closures show a similar trend with both numerical models comparing well to experiments (see Figure 9). Steady state flow rates were accurately predicted for most partial closure experiments after valve closure but were generally overestimated with errors $\sim 20 \%$. RC and MOC models also perform relatively well for $89 \%$ valve closures (Figures 10 and 11). The peak pressure predictions were reasonably accurate with small deviations more than likely caused by experimental inconsistency. The numerical models accurately predicted steady state pressures for the majority of cases, with errors generally $<30 \%$.

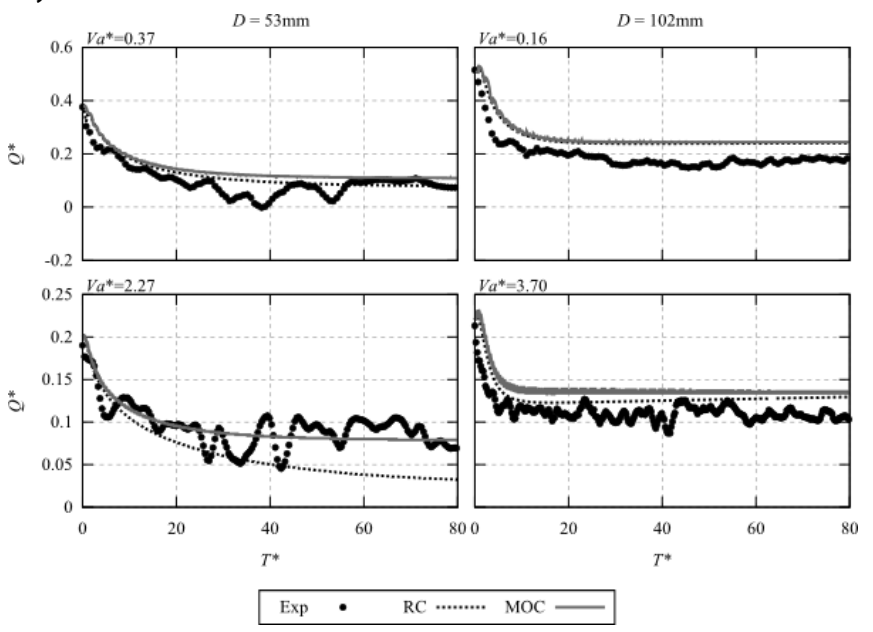

Figure 9 Flow rate hydrographs for $81 \%$ valve closures.

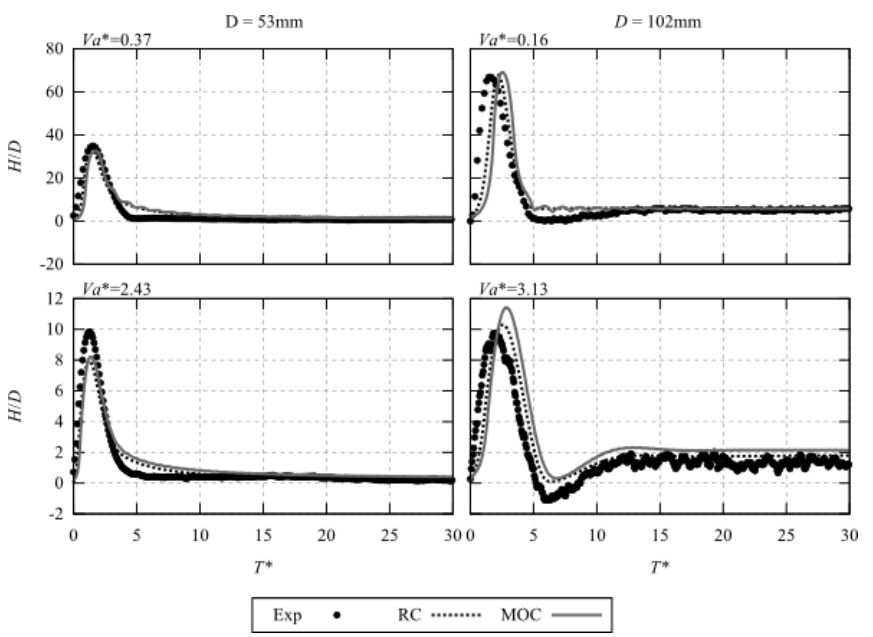

Figure 10 Pressure hydrographs for $89 \%$ valve closures.

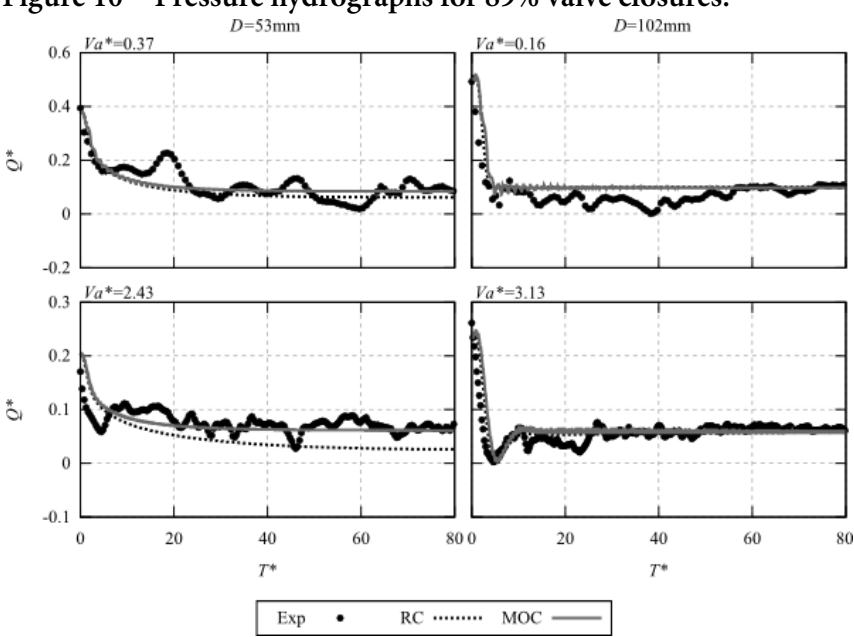

Figure 11 Flow rate hydrographs for $89 \%$ valve closures. 
Flow rate predictions showed a similar accuracy for $89 \%$ valve closures and $81 \%$ closures. Average errors for steady state discharge predictions ( $89 \%$ closures) were $\leq 30 \%$. As noticed in $81 \%$ closures, the numerical model performance was comparable for both pipe diameters with $89 \%$ valve closures.

Maximum and minimum pressure results for partial valve closures are presented in Figure 12. As shown in Figures 8 and 10, $\mathrm{RC}$ and MOC peak pressure predictions have similar accuracy. The MOC model tends to predict larger pressures than the RC model for $D=53 \mathrm{~mm}$ experiments and smaller pressures for $D=102 \mathrm{~mm}$ experiments. Both models perform fairly well with maximum errors $<20 \%$ and average errors $\sim 10 \%$.
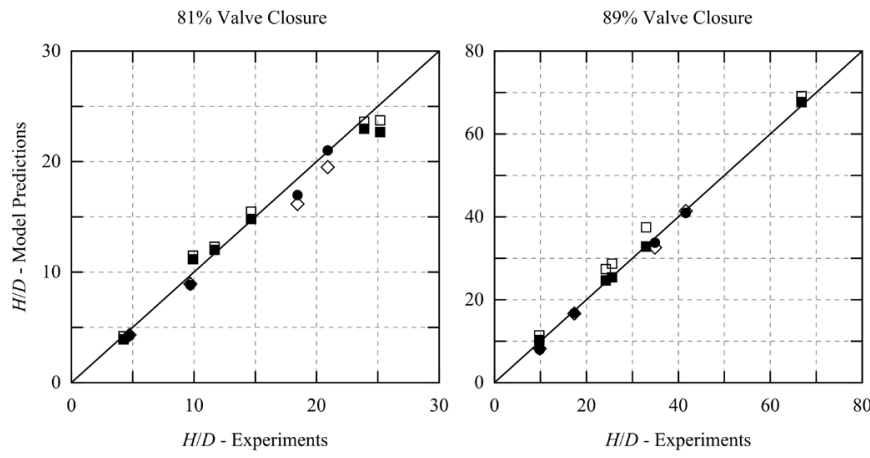

RC: $D=53 \mathrm{~mm} \quad \bullet \quad$ RC: $D=102 \mathrm{~mm} \quad$ - $\quad$ MOC: $D=53 \mathrm{~mm} \quad \diamond \quad$ MOC: $D=102 \mathrm{~mm}$

Figure 12 Peak pressure predictions compared to experiments for partial valve closures.

\section{Conclusions}

Surges caused by sudden air pocket compression were studied in this work for $D=53 \mathrm{~mm}$ and $D=102 \mathrm{~mm}$ PVC pipelines in adverse slope conditions. The experiments expanded on the work of Vasconcelos and Leite (2012), who tested a wide range of slopes in $\mathrm{D}=102 \mathrm{~mm}$ pipes. Pressures were supplied by an upstream reservoir in which pressurized flow existed at the upstream end of a $10.7 \mathrm{~m}$ or $12.0 \mathrm{~m}$ pipeline and free surface flow at the downstream end. The sudden closure of a downstream valve initiated the surge event trapping an air pocket of measured volume. A large range of air pocket volumes and flow rates were tested for $81 \%, 89 \%$ and $100 \%$ valve closures.

Lumped inertia $(\mathrm{RC})$ and discretized (MOC) numerical models were developed and tested for a wide range of experiments. The objective was to determine the viability of each numerical model focusing on peak surge pressures, flow rates and the pipeline $L / D$ ratio. For total valve closures, both numerical models consistently overestimated peak pressure magnitudes (minimum and maximum) regardless of the experimental condition. The predicted maximum pressures were reasonably accurate for experiments with smaller flow rates or larger air pocket volumes and thus smaller normalized pressures $(H / D)$. The accuracy of both numerical models decreased for experiments with large flow rates, largely because of difficulties in measuring the smaller air pocket volumes. The maximum pressure predictions started to diverge from experiments and $\mathrm{MOC}$ model predictions when $H / D>40$. Significant discrepancies were observed for rigid column results when maximum $H / D>60$ to 80 . Thus, for the geometries tested in this work, the rigid column model should be used with caution when $H / D>40$ or $V_{a}^{*}<1$ and is not recommended when peak pressure head ratios exceed this range.

The results for negative pressures did not exactly mirror maximum pressure results. $\mathrm{RC}$ and $\mathrm{MOC}$ model predictions compared well to one another for the range of experiments presented in this work. However, $|H / D|$ values for negative pressures were never $>40$, so additional experiments are needed to determine if the rigid column model diverges from experiments when negative pressure head ratios approach 80 as seen for maximum pressure results.

Inertial frequencies were resolved with good accuracy for experiments with smaller flow rates and larger air pocket volumes. However, these results significantly diverged for cases with small air pocket sizes $\left(V_{a}^{*}<1\right)$, which may have been caused by the difficulty in measuring these small volumes of air. A key limitation for both numerical models was pressure damping predictions, which was significantly underestimated for total valve closure cases. The MOC model performed noticeably better than the RC model in predicting this decay in pressures, but both models did not perform well in this regard. The source of these energy losses remains an important and open problem in hydraulics (Vasconcelos et al. 2011; Vasconcelos and Leite 2012).

Peak pressures were better predicted for partial valve closure cases in which numerical model predictions were accurate throughout the range of flow conditions tested. The numerical model accuracy depended on calibrating the discharge coefficient at the downstream valve with experiments as done in Vasconcelos and Leite (2012) and thus the system geometry should be carefully considered. For partial valve closures, negative pressures were minimal or non-existent and the spring-mass feature observed for total valve closures did not occur (Vasconcelos and Liete 2012).

As was noted in Malekpour and Karney (2014), the RC model has a limitation for large $L / D$ ratios that depends on the air pocket volume, acoustic wave speed and kinetic energy upstream of the air pocket. In this work $L / D$ ratios of 118 and 223 were tested with $V_{a}^{*}$ values ranging from 0.3 to 3.7. For both $L / D$ ratios tested, RC model predictions diverged from the MOC model at approximately the same maximum $H / D$ value. However, these error magnitudes varied between experiments with different pipe diameters, particularly for cases with larger pressures. More than likely, the error discrepancies were caused by different slopes used for $D=53 \mathrm{~mm}$ and $D=102 \mathrm{~mm}$ experiments, thus effecting the air pocket volumes. The system geometry is clearly important highlighting the need for additional experimental work as indicated in Vasconcelos and Leite (2012) to isolate the $L / D$ ratio effects. Results presented in this work provide insight into the viability of the $\mathrm{RC}$ model based on peak $H / D$ ratios and air pocket 
sizes. Unfortunately, these parameters may be difficult to obtain for the practicing engineer. Additional surge experiments are needed to better assess viable modeling alternatives based on a wider range of geometrical parameters.

\section{Acknowledgments}

The authors would like to acknowledge the support provided by LimnoTech Inc., and by Auburn University, which has funded part of this research.

\section{References}

Chaudhry, M. H. 1987. Applied Hydraulic Transients. New York: Van Nostrand Reinhold.

De Martino G., N. Fontana and M. Giugni. 2008. “Transient Flow Caused by Air Expulsion through an Orifice." Journal of Hydraulic Engineering 134 (9): 1395-9.

Lee, N. H. 2005. Effect of Pressurization and Expulsion of Entrapped Air in Pipelines. Atlanta: Georgia Institute of Technology. Doctoral dissertation.

$\mathrm{Li}$, J. and A. McCorquodale. 1999. "Modeling Mixed Flow in Storm Sewers." Journal of Hydraulic Engineering 125 (11): 1170-80.

Malekpour, A. and B. W. Karney. 2014. “Discussion of 'Pressure Surges Following Sudden Air Pocket Entrapment in Storm-Water Tunnels' by José G. Vasconcelos and Gabriel M. Leite." Journal of Hydraulic Engineering 140 (4): 1081-9.

Martin, C. S. 1976. "Entrapped Air in Pipelines." In Proceedings of the Second International Conference on Pressure Surges, edited by H. S. Stephens, A. L. King and C. A. Stapleton. Cranfield, Bedfordshire: BHRA.

Pozos O., C. A. Gonzalez, J. Giesecke, W. Marx and E. A. Rodal. 2010. "Air Entrapped in Gravity Pipeline Systems." Journal of Hydraulic Research 48 (3): 338-47.
Press W., B. Flannery, S. Teukolsky and W. Vetterling. 1989. Numerical Recipes in Pascal: The Art of Scientific Computing. Cambridge: Cambridge University Press.

Reddy H. P., M. H. Chaudhry and P. K. Mohapatra. 2010. “Modeling of Periodic Flows in Pipelines by Transfer Function Method." Journal of Hydraulic Research 48 (2): 255-9.

Soares A. K., D. I. Covas and L. F. Reis. 2008. "Analysis of PVC PipeWall Viscoelasticity During Water Hammer." Journal of Hydraulic Engineering 134 (9): 1389-94.

Vasconcelos, J. G. and G. M. Leite. 2012. "Pressure Surges Following Sudden Air Pocket Entrapment in Stormwater Tunnels." Journal of Hydraulic Engineering 134 (7): 984-92.

Vasconcelos, J. G. and S. J. Wright. 2006. “Improved Simulation of Flow Regime Transition in Sewers: The Two Component Pressure Approach." Journal of Hydraulic Engineering 132 (6): 553-62.

Vasconcelos, J. G. and S. J. Wright. 2009. "Investigation of Rapid Filling of Poorly Ventilated Stormwater Storage Tunnels." Journal of Hydraulic Research 47 (5): 547-58.

Vasconcelos, J., Wright, S., and Lautenbach, D. 2011. “Modeling Approaches for the Rapid Filling of Closed Conduits with Entrapped Air". World Environmental and Water Resources Congress 2011: pp. 3449-3458. doi: 10.1061/41173(414)362

Wylie, E. B. and V. L. Streeter. 1993. Fluid Transients in Systems. Englewood Cliffs, NJ: Prentice-Hall.

Zhou F., F. E. Hicks and P. M. Steffler. 2002. “Transient Flow in a Rapidly Filling Horizontal Pipe Containing Trapped Air." Journal of Hydraulic Engineering 128 (6): 625-34.

Zhou L., D. Liu, B. Karney and Q. Zhang. 2011. “Influence of Entrapped Air Pockets on Hydraulic Transients in Water Pipelines." Journal of Hydraulic Engineering 137 (12): 1686-92. 